Gut and Liver, Vol. 10, No. 4, July 2016, pp. 502-508

\title{
Navigating the Microbial Basis of Inflammatory Bowel Diseases: Seeing the Light at the End of the Tunnel
}

\author{
Laura E. Raffals ${ }^{1}$ and Eugene B. Chang $^{2}$ \\ ${ }^{1}$ Division of Gastroenterology and Hepatology, Mayo Clinic, Rochester, MN, and ${ }^{2}$ Division of Gastroenterology and Hepatology, University of \\ Chicago, Chicago, IL, USA
}

Inflammatory bowel diseases (IBD), such as Crohn's disease and ulcerative colitis, are chronic inflammatory conditions affecting the gastrointestinal tract with variable presentations and disease courses. The cause of IBD is unknown, but it is hypothesized that individuals with a genetic predisposition to disease develop an aberrant immune response to environmental triggers. Evidence suggests that microbiota residing in the gastrointestinal tract play an important role in the development and perpetuation of the disease. In this review, we discuss the role of microbes in the development of a healthy gut, their role in the development of diseases in general, and their role in the development of IBD. Advances in molecular technologies and bioinformatics will continue to further our insight into the structure of the microbial community, the function of the microbial community as a whole, and the interaction of this community with the host immune system. The latter two are crucial to understanding the role of microbes in IBD. The field has advanced significantly in recent years, and the future is very promising as we begin to elucidate the microbial basis of IBD. (Gut Liver 2016;10:502508)

Key Words: Inflammatory bowel disease; Crohn disease; Colitis, ulcerative; Microbiota; Microbial

\section{INTRODUCTION}

The inflammatory bowel diseases (IBD) are a group of heterogeneous disorders involving the gastrointestinal tract affecting more than 3.6 million people in the United States and Europe. ${ }^{1}$ There are two primary clinical phenotypes of IBD, ulcerative colitis (UC) and Crohn's disease (CD). UC involves only the colon and the inflammatory pattern is characterized by a superficial inflammation beginning at the anal verge and extending proximally through the colon in a continuous manner. The extent of inflammation can vary amongst patients. CD can involve any segment of the gastrointestinal tract (from the mouth to anus), can be patchy in nature, and in some cases is a deep inflammatory process that can lead to complications such as fibrosis or stricturing of the intestine or the development of fistulas to other loops of intestine or other organs (such as the bladder or skin). Most patients are diagnosed in their late teen years or twenties. There appears to be a genetic predisposition to these diseases, although most patients do not have a family history of IBD, highlighting the importance of environmental risk factors for the development of disease.

The cause of IBD is not known, but most experts agree that IBD develops in the setting of a genetic predisposition to the disease, exposure to an environmental trigger, and a subsequent aberrant immune response resulting in disease. Gut microbes are important components in this etiopathogenesis mix, either by providing antigenic stimuli or through alterations in membership and function that promote host-microbe imbalances that disturb intestinal and immune homeostasis. Microbes may also be mediators of environmental triggers of IBD; however, this aspect of the IBD story is still unraveling.

In this conceptual review, we will cover the role of the microbiome and health; microbiome and disease; microbiome and IBD and finally the future of microbiome research in IBD.

\section{THE ROLE OF GUT MICROBES IN DEVELOPMENT AND MAINTENANCE OF HEALTH}

Soon after we are born, we acquire microbes from our mothers, the environment, and things (animate and inanimate) that we encounter which become the building blocks of our micro-

Correspondence to: Eugene B. Chang

Department of Medicine, Knapp Center for Biomedical Discovery, University of Chicago, 900 E. 57th Street, Chicago, IL 60637, USA

Tel: +1-773-702-6458, Fax: +1-773-702-2281, E-mail: echang@medicine.bsd.uchicago.edu

Received on August 24, 2015. Accepted on December 2, 2015.

pISSN 1976-2283 eISSN 2005-1212 http://dx.doi.org/10.5009/gnl15491

@) This is an Open Access article distributed under the terms of the Creative Commons Attribution Non-Commercial License (http://creativecommons.org/licenses/by-nc/4.0) which permits unrestricted non-commercial use, distribution, and reproduction in any medium, provided the original work is properly cited. 
biome. In the gut, the process proceeds organically-influenced by factors like host genetics, diet, and a competitive selection among microbes themselves that ultimately evolves into a syntrophic relationship among community members as well as with the host. This formative period of gut microbiome development is extremely important for the proper development of host organismal functions that includes the gut, but also other extraintestinal organ systems. The proper development of gut microbiota with sufficient diversity within the first few years of life $^{2}$ is also likely key to the ontogenesis of immune and metabolic networks. Perturbations in the proper development of the gut microbiome can therefore have long-term consequences and account for the ever increasing and alarming trends in complex immune disorders and metabolic diseases that have occurred over the past few decades. ${ }^{3-5}$ While the notion of developing a "healthy" gut microbiome in the early stages of life is becoming increasingly accepted, the conundrum of what a "healthy" microbiome is remains. It is clear from many studies that the gut microbiome among healthy adult individuals varies tremendously. ${ }^{6-8}$ What is a "healthy" microbiome in one individual may not be healthy in another. Consequently, any attempt to reshape the gut microbiome in early life is problematic without knowing what the best end point is for individual subjects. Nonetheless, most accept the paradigm that the natural development of a diverse gut microbiota, i.e., one that has a membership comprised of many types of microbial species, is best. On the other end of the spectrum are extreme examples of what happens when there is limited or no diversity of the gut microbiome. Hospitalized or institutionalized patients who are placed on broad spectrum antibiotics for extended periods are at increased risk for the development of antibiotic-associated colitis, a condition that arises when there is loss of diversity and abundance of most commensal microbiota that favors the bloom an opportunistic pathogenic microbe, Clostridium difficile. ${ }^{9,10}$ In germ-free mice that are brought up in a sterile environment and lack any microbiomes, profound immaturity of many organs systems can be observed along with developmental anomalies of immune and metabolic homeostasis. Thus, these examples illustrate the pivotal role that the gut microbiome plays in health and in the development of disease.

\section{THE ROLE OF GUT MICROBES IN DEVELOPMENT OF DISEASE}

Immune-mediated diseases, such as IBD, asthma, type 1 diabetes, and multiple sclerosis are most prevalent in industrialized countries and are rare in less-developed countries. In recent decades, epidemiological studies have observed an increase in autoimmune diseases in industrialized countries. Environmental factors are likely responsible for this increased incidence and many believe that a simultaneous decrease in infectious agents and increase in personal cleanliness may be linked to the trend of increased autoimmune conditions. Proponents of this theory believe that children who are not exposed to infectious agents, commensal organisms and parasites, fail to develop immune tolerance to antigens leading to increased susceptibility to autoimmune diseases (Fig. 1). Improved personal hygiene, pasteurization of food, and increased exposure to antibiotics while
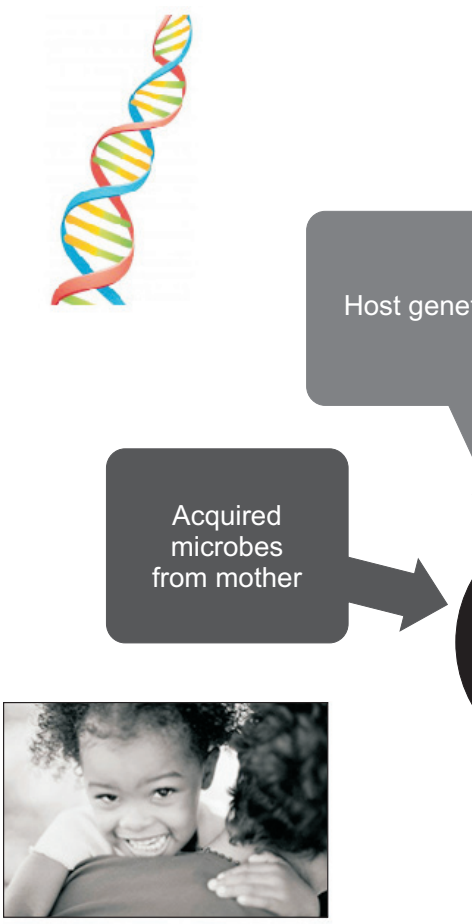

Diet

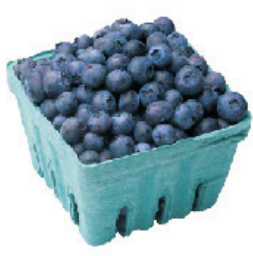

Competitive selection amongst microbes

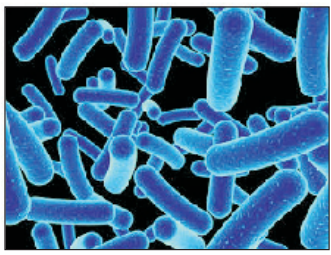

Fig. 1. Factors influencing the gut microbiome after birth. Many genetic and environmental factors influence the infant gut microbiome. Exposure to the mother's microbiome, beginning with the birthing process, and an infant's genetic background, diet and competitive selection among the microbes themselves are all important factors for the development of the microbiome. 
seemingly indicative of advances of modern society, may have unintended consequences to the developing immune system (Table 1).

Microbes appear to be necessary for the development of many autoimmune diseases. Murine models have demonstrated the need for bacteria to trigger disease states. In IBD, rheumatoid arthritis and multiple sclerosis murine models, germ-free mice remain disease-free. ${ }^{2,11}$

Epidemiological studies have shown that heavy use of antibiotics during childhood is a risk factor for a variety of autoimmune conditions (type 1 diabetes, atopy, juvenile idiopathic arthritis, and inflammatory bowel disease. ${ }^{12-16}$ Antibiotics are the most commonly prescribed medication in children accounting for one fourth of all prescriptions written in the pediatric population ${ }^{17}$ although studies have suggested that many antibiotic prescriptions for children are unnecessary. ${ }^{18,19}$ Antibiotics disturb the gut microbiota decreasing microbial diversity, and in theory may disturb the balance of commensal and disease promoting organisms. While microbial communities appear to recover to some degree after completion of antibiotic therapy, there can be permanent compositional changes of the community. ${ }^{20}$ The health consequences of these compositional changes are an area of great interest. Although still supported by limited data, it is possible that antibiotic exposure during early childhood has significant influence on the gut microbial community through the elimination of key taxa opening the door for pathogenic organisms and pathobionts to bloom which may have consequences on the development of the host immune system.

Exposure to some microbes is believed to protect against disease states. For example, helminth exposure has been postulated as a protective environmental factor. IBD and other immunemediated diseases are uncommon is less-developed countries where most people are infested with worms. ${ }^{21}$ Observational studies have demonstrated a modulation of inflammation in helminth-infested individuals with immune-mediated diseases. ${ }^{22}$ Investigators in Germany reported the case of a 12-year-old girl with UC and Enterobius vermicularis infection whose colitis was latent during worm infestation, but activated following worm clearance. During worm infestation mucosal regulatory T cells (Treg) were increased, based on expression of the Foxp3 tran-

Table 1. Factors Reducing Microbial Exposure in the Modern World

Hygiene theory: factors reducing microbial exposure

Improved personal hygiene

Pasteurization of food

Antibiotics

Absence of helminth infestation

Caesarean section

Less crowded living environments

Antibacterial cleaning products scription factor and increased mRNA levels for immunomodulatory cytokines, IL-10 and transforming growth factor $\beta$ (TGF- $\beta$ ) that were significantly reduced following worm clearance. ${ }^{22}$ Animal studies have shown that helminths blunt both Th1 and Th2 immune responses and foster an immunoregulatory immune response through Treg, IL-10 and TGF- $\beta .^{23}$ In murine models of IBD, helminth eggs and live worms have also been shown to protect against the development of inflammation. ${ }^{23,24}$ This protective effect is likely due to this enhanced immunoregulatory immune response (Treg).

\section{THE ROLE OF THE GUT MICROBES IN DEVELOPMENT AND OUTCOMES OF INFLAMMATORY BOWEL DISEASE}

IBD is an example of a Western disorder arising from the "perfect storm" of environmental, genetic, and microbial factors. In most cases, none of these factors is sufficient to result in disease, however in the presence of several of these factors, susceptibility to disease rises. Human genetic studies of IBD including more than 75,000 cases and controls have identified nearly 200 host susceptibility loci. ${ }^{25,26}$ Analyses of these loci suggest that the host-microbe interaction is crucial in the development of disease. In health, the intestinal mucosal immune system reacts to pathogenic antigens while maintaining tolerance to commensal organisms. In IBD, it appears that something in the environment triggers the host immune system to lose tolerance to components of commensal organisms.

There is little debate that the enteric microbiome plays an integral role in IBD. Even prior to the advances in molecular technology allowing us to deeply explore the relationship of the gut microbial communities and IBD, several observations supported the theory that microbes have a critical role in the development of IBD. D'Haens et al. ${ }^{27}$ reported their findings in 1998 that infusion of fecal contents triggered a postoperative recurrence of ileal CD. Other evidence supporting the role of gut microbes in IBD include the clinical observation that patients often report antibiotic exposure or infections prior to IBD onset, the presence of serological markers reflecting "leaky" guts and immunologic reactions to organisms in patients with IBD, and the attenuation of inflammation in animal models of IBD in germ-free environments.

What is not well understood is how alterations in the gut microbiota lead to or perpetuate the inflammatory response in IBD. It is believed that IBD occurs in genetically susceptible individuals when the balance between host and microbial factors is lost. The mucosal immune system of the gastrointestinal tract is an important barrier to potential pathogens. The intestinal epithelial and immune cells come in contact with foreign material and are tasked to respond to potential pathogens, mounting an inflammatory response to eliminate or contain the offending agents, protecting the host. The mucosal immune system must also recognize environmental factors (dietary and microbial) 
which are not pathogenic, mounting a subclinical inflammatory response of tolerance. If this system is broken, the host is either susceptible to infection or in the setting of an exacerbated immune response, chronic inflammation such as that seen in IBD. Supporting the role of loss of tolerance mechanisms in IBD, patients with IBD have shown to exhibit mucosal secretion of IgG antibodies against commensal bacterial as opposed to IgA antibodies, the latter of which is the normal physiological response which does not induce inflammation. ${ }^{28}$ Alternatively, altered microbial communities and host response to these communities can lead to a vicious cycle of intestinal dysbiosis leading to immune activation and inflammation with resultant suppression of the healthy microbiota and selection of pathobionts, leading to an inflammatory disorder.

Early studies of IBD and the microbiome utilized low-resolution surveys of the enteric microbial communities, however in recent years many investigators employ next-generation sequencing technology allowing for a deeper description of microbial communities and their function. Making sense of the results of these many studies has been daunting. Studies produce an incredible amount of data, are often collected in a variety of patient subsets, in a cross-sectional manner in which other factors such as medications may have important confounding effects. The sample studied in these studies also varies and can include stool or luminal samples or mucosal biopsies.

Although there are recognized limitations to the existing data, we have learned a great deal about the role of the gut microbiota in IBD. In the gut of IBD patients, there appears to be a decrease in $\alpha$-diversity (species richness) and decreases in taxa within Bacteroides and Firmicutes while Gammaproteobacteria is increased. ${ }^{29}$ Within the Clostridia class, there is also a shift in genera in patients with CD. Roseburia and Faecalibacterium are decreased while Ruminococcus gnavus is increased. Faecalibacterium prausnitzii, a bacterium of particular interest in IBD, is one of the most abundant organisms in the enteric microbiota, but is decreased in mucosal ileal biopsies from patients with $\mathrm{CD}$ and in patients at higher risk of postoperative recurrence of CD. ${ }^{30}$ Low counts of $F$. prausnitzii in patients with UC were also associated with less than 12 months of remission and more than 1 relapse per year. ${ }^{31}$ Additional evidence of dysbiosis in UC patients includes studies reporting an increase in Deltaproteobacteria, which contains many of the sulfate-reducing bacteria. ${ }^{32}$

A recent study collecting samples from children recently diagnosed with IBD and naïve to treatment found abundance of Enterobacteriaceae, Pasteurellacaea, Veillonellaceae, and Fusobacteriaceae, and decreased abundance of Erysipelotrichales, Bacteroidales, and Clostridiales. These community changes correlated with increased disease activity. ${ }^{33}$ Exposure to antibiotics was also noted to exacerbate the community dysbiosis.

In order to understand the role of the microbiota in IBD, one must go beyond consideration of microbial composition and consider the functional diversity of the enteric microbiota (Fig.
2). The microbiome is vital to maintaining host health and is involved in many processes including influencing immune system development, energy metabolism, drug metabolism, and vitamin biosynthesis. Morgan et al..$^{34}$ performed a study with the intent to bridge the gap between studies examining composition and functional differences of the gut microbiota in IBD and healthy individuals while accounting for environmental factors such as age, treatment, and tobacco use. These investigators assessed the composition of the microbial communities of the gut and further analyzed the inferred metagenome using reference genomes. This inferred functional metagenomic analysis found shifts in the microbial community in patients with IBD as previously described (changes in Firmicutes and Proteobacteria phyla), however they noted that there were functional themes in the observed dysbiosis and changes in function appeared to be more common than change in composition alone. Twelve percent of metabolic pathways were differentially abundant in patients with IBD compared to controls while only $2 \%$ of genera were significantly different between populations. Their observed changes in microbiome function may possibly reflect the microbiomes response to oxidative stress and nutrient availability in the setting of inflammation. There were also changes in carbohydrate metabolism and amino acid biosynthesis. Ileal $\mathrm{CD}$ was associated with an increase in virulence and secretion pathways. $^{34,35}$

One of the important functions of the microbiome is the production of short chain fatty acid (SCFA), acetate, propionate, and butyrate. Bacteria ferment dietary fiber producing SCFAs which are a primary energy source for the colonic epithelium and may induce expansion of $\mathrm{T}$ regulatory cells. ${ }^{36}$ The bacteria that produce SCFAs are phylogenetically diverse however there are many examples of bacterial groups known to pro-

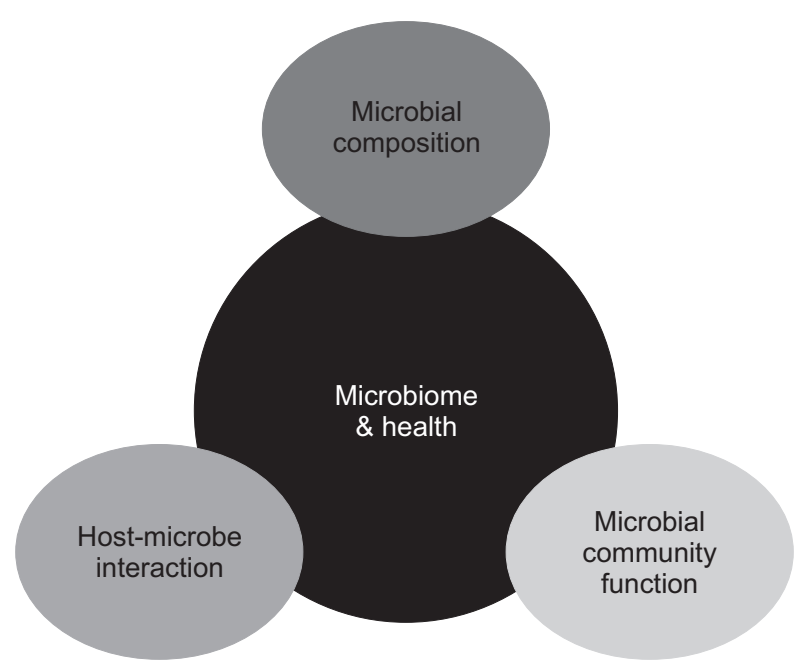

Fig. 2. The role of the gut microbiome in inflammatory bowel diseases. Although the microbial composition of the gut microbiota is important for health, recent studies have recognized the importance of the function of the microbial community and the host-microbial interaction in health and disease. 
duce SCFAs that appear to be decreased in IBD. Roseburia and Phascolarctobacterium are decreased in CD while Odoribacter and Leuconostocaceae are decreased in UC. ${ }^{34}$ Faecalibacterium, which has been associated with IBD as described above, is another SCFA-producing bacteria. Metagenomic studies of the enteric microbiome have also shown a decrease in genes related to butyrate and propionate metabolism in CD patients. ${ }^{34}$

In addition to function, investigators are also beginning to consider the role of the host-microbial interaction in IBD. A recent study of UC and FAP patients with ileal pouches compared host gene expression and the mucosal microbiome in patients with and without pouchitis. ${ }^{35}$ Host gene expression in UC patients varied based on sampling location (prepouch vs pouch). However, the microbial community was not significantly different in the prepouch ileum compared to the pouch suggesting host gene expression does not determine microbial composition. Antibiotic use had the greatest effect on microbial composition, while inflammation was associated with changes in host gene expression. While the cross-sectional nature of this study did not allow for determination of causation of inflammation, this study moves the field forward in gaining biological insights into the complex interactions of host and microbial community in disease.

\section{RESTORING BALANCE OF THE GUT MICROBIAL COMMUNITY}

Most treatments for IBD counteract the host immune response driving the inflammatory process. With the greater appreciation of the role of the microbial community in disease, there is an interest to restore the balance of the gut microbial community through diet, prebiotics, probiotics, and fecal microbial transplantation (FMT). Historically, antibiotics have been the most frequently used treatment to alter the gut microbiota; however this mode of treatment has met limited success with the exception of treatment of pouchitis. Prebiotics are poorly digested carbohydrates (fiber) that are selectively fermented by bacteria and enhance growth of commensal microbes. Probiotics are beneficial live microorganisms aimed to restore the healthy microbes in the gut microbial community. While prebiotics and probiotics are different forms of microbial therapy, both aim to restore balance of the microbiota. These supplements are not regulated by the Food and Drug Administration (FDA) and not subject to quality control measures. Given this limitation, their effects are likely inconsistent and difficult to study. It is unlikely that either of these treatment modalities is sufficient to provide a lasting and meaningful treatment response in IBD patients, particularly if given as monotherapy.

There is more enthusiasm for FMT in the treatment of IBD. FMT is believed to have the potential to restore the normal gut flora and has had great success in the treatment of recurrent $C$. difficile infection which has led to interest using FMT to restore the underlying dysbiosis found in IBD. The first report of FMT for the treatment of IBD was in 1989, when one of the paper's authors described use of FMT (given as retention enemas) to treat his UC. He described histologic remission at 3 months and clinical remission at 3 and 6 months. ${ }^{37}$ There have been mixed results in subsequent small clinical trials examining FMT in UC which is likely a result of small sample sizes, variation in methodology and selection of donor stool. Recently a systematic review and meta-analysis looked at 18 studies including 122 patients with IBD who underwent FMT. Clinical remission was achieved in $45 \%$ of patients, and $36.2 \%$ when excluding case series. ${ }^{38}$ Two recent studies of FMT for UC were recently reported, both of which were stopped prematurely due to futility. ${ }^{39,40}$ However, in one of these trials, remission was achieved in (9/38) $24 \%$ of patients receiving FMT compared to (2/37) 5\% of patients receiving placebo, meeting the primary endpoint of the trial. ${ }^{39}$ The role of FMT in IBD is not yet known. The inconsistent results in UC trials and the limited data available for CD make it difficult to speculate if FMT will be a successful treatment we can offer our patients. Larger trials are needed, and optimized methods for administration including duration of treatment need to be elucidated. There is great interest to explore methods of manipulating the gut microbiota in IBD and hopefully in the future we will have a greater understanding how best to address the microbial dysbiosis often encountered in IBD.

\section{THE FUTURE: OVERCOMING CHALLENGES AND MOVING FORWARD}

IBD is a group of complex diseases that has great variability amongst the individuals it affects. The hope of the IBD research community is to eventually cure and prevent this group of diseases. However, before we can reach a cure, we must understand the barriers preventing us from achieving this ambitious goal (Table 2). The first step in advancing our efforts to a cure

Table 2. The Future of Microbial Ecology and Inflammatory Bowel Diseases

Comprehensive phenotyping of patients, allowing personalized approach to therapy

Genetic, serologic, microbial signatures

Team approach to discovery and application, bridging the gap between clinician and scientist

Investment in longitudinal studies of the microbiome and IBD

Moving beyond descriptive studies of microbiota, incorporating microbial function and role of the host-microbe interaction 
is to recognize that we must individualize our approach to each patient suffering from these diseases. There is likely not one cause of IBD. Rather the etiopathogenesis is likely variable amongst affected individuals. As a result our approach to treating our patients must be individualized as well. We propose the phenotyping of patients should include not only the extent and nature of disease, but also the genetic, serologic and microbial characteristics as well. In order to integrate these data into meaningful datasets, we must overcome the disconnect between clinicians and researchers, each seemingly looking at the same problem in isolation. A team approach leading to better communication, education and information distribution could lead to the development of diagnostic and management algorithms integrating clinical metadata and new technologies. To achieve this, clinicians must help researchers understand the reach and impact of their work. Once a microbial ecologist understands how their work can translate into improving the lives of many patients, their work becomes even more meaningful. Alternatively, researchers need to help clinicians understand the technology and tools that are readily used in the field and how to make sense of latest discoveries. Often clinicians are intimidated by basic research, however as the dialog improves, a team approach has the opportunity to move the field of IBD forward at a much faster pace.

Second, we need to learn to make best use of the technologies available to us. There has been a rapid evolution of technology in the sequencing field. This rapid evolution has exceeded our abilities to understand how best to use these technologies and analyze and integrate massive data sets. Through the excitement of available platforms, investigators have in essence jumped the gun; acquiring data without a clear purpose or plan for interpretation of findings. This has resulted in an accumulation of a large amount of data that is either uninterpretable or questionably interpreted. To address this issue, it is important that leaders in the field help develop standard operating procedures for sample acquisition and processing. Scientists must move beyond the frame of mind intent to biobank, collect samples and process. Instead, we must think through the questions we aim to answer and plan our studies accordingly. We must think through our study designs to achieve a top-down approach. Many studies of the microbiome are cross-sectional in nature, however longitudinal studies are required to understand the cause-effect relationship of changes in the microbiome and disease. The sampling method must also be carefully considered. For example, in IBD (a disease of the gut mucosa) should we study intestinal mucosal samples or luminal samples as we address questions of the microbiome and disease? The effect of colonic lavage on the microbiota must be considered. Finally, communication and teamwork are crucial such that the members of the team formulating the biological question and those analyzing the data are able to produce results in the appropriate context understanding the important confounding variables which might include disease specific factors such as medications, history of surgical resection, diet and smoking status.

If the ultimate goal is to prevent and cure IBD, we must take a step back and calculate how we predict risk of disease or flare of disease. This may involve identifying those individuals genetically predisposed to disease and learning how reshaping the gut microbiome leads to disease or flare of disease. In order to finally cure IBD, we should first identify homogenous sets of patients where the etiopathogenesis is known. This is occurring in familial exome sequencing studies where families with a high penetrance of disease are studied, patients phenotyped and a common IBD susceptibility gene identified. The function of this genetic variant can be studied and targeted for individualized treatment. This approach allows us to direct therapies to specific targets and pathways whether directed at the microbial community or the host.

This is an exciting time in the IBD research community. Investigators across institutions are working together to study biologically relevant questions in IBD at a time when technological advances in microbiome research and the growing field of computational biology might allow us to finally understand how to provide precision medicine for our IBD patients who have already suffered enough.

\section{CONFLICTS OF INTEREST}

No potential conflict of interest relevant to this article was reported.

\section{ACKNOWLEDGEMENTS}

Source of funding: NIDDK grants P30DK42086, DK097268, and T32DK07074, Leona and Harry Helmsley Trust, Gastrointestinal Research Foundation of Chicago.

\section{REFERENCES}

1. Loftus EV Jr. Clinical epidemiology of inflammatory bowel disease: incidence, prevalence, and environmental influences. Gastroenterology 2004;126:1504-1517.

2. Lee YK, Mazmanian SK. Has the microbiota played a critical role in the evolution of the adaptive immune system? Science 2010;330:1768-1773.

3. Devkota S, Wang Y, Musch MW, et al. Dietary-fat-induced taurocholic acid promotes pathobiont expansion and colitis in II10-/mice. Nature 2012;487:104-108.

4. Dalal SR, Chang EB. The microbial basis of inflammatory bowel diseases. J Clin Invest 2014;124:4190-4196.

5. Arrieta MC, Stiemsma LT, Amenyogbe N, Brown EM, Finlay B. The intestinal microbiome in early life: health and disease. Front Immunol 2014;5:427.

6. Human Microbiome Project Consortium. Structure, function and 
diversity of the healthy human microbiome. Nature 2012;486:207214.

7. Dominguez-Bello MG, Blaser MJ, Ley RE, Knight R. Development of the human gastrointestinal microbiota and insights from highthroughput sequencing. Gastroenterology 2011;140:1713-1719.

8. Morgan XC, Segata N, Huttenhower C. Biodiversity and functional genomics in the human microbiome. Trends Genet 2013;29:51-58.

9. Hookman P, Barkin JS. Clostridium difficile associated infection, diarrhea and colitis. World J Gastroenterol 2009;15:1554-1580.

10. Theriot CM, Young VB. Microbial and metabolic interactions between the gastrointestinal tract and Clostridium difficile infection. Gut Microbes 2014;5:86-95.

11. Wu HJ, Wu E. The role of gut microbiota in immune homeostasis and autoimmunity. Gut Microbes 2012;3:4-14.

12. Johnson CC, Ownby DR, Alford SH, et al. Antibiotic exposure in early infancy and risk for childhood atopy. J Allergy Clin Immunol 2005;115:1218-1224.

13. Kilkkinen A, Virtanen SM, Klaukka T, et al. Use of antimicrobials and risk of type 1 diabetes in a population-based mother-child cohort. Diabetologia 2006;49:66-70.

14. Arvonen M, Virta LJ, Pokka T, Kröger L, Vähäsalo P. Repeated exposure to antibiotics in infancy: a predisposing factor for juvenile idiopathic arthritis or a sign of this group's greater susceptibility to infections? J Rheumatol 2015;42:521-526.

15. Virta L, Auvinen A, Helenius H, Huovinen P, Kolho KL. Association of repeated exposure to antibiotics with the development of pediatric Crohn's disease: a nationwide, register-based Finnish case-control study. Am J Epidemiol 2012;175:775-784.

16. Shaw SY, Blanchard JF, Bernstein CN. Association between the use of antibiotics and new diagnoses of Crohn's disease and ulcerative colitis. Am J Gastroenterol 2011;106:2133-2142.

17. Hicks LA, Taylor TH Jr, Hunkler RJ. U.S. outpatient antibiotic prescribing, 2010. N Engl J Med 2013;368:1461-1462.

18. McCaig LF, Besser RE, Hughes JM. Antimicrobial drug prescription in ambulatory care settings, United States, 1992-2000. Emerg Infect Dis 2003;9:432-437.

19. Nash DR, Harman J, Wald ER, Kelleher KJ. Antibiotic prescribing by primary care physicians for children with upper respiratory tract infections. Arch Pediatr Adolesc Med 2002;156:1114-1119.

20. Dethlefsen L, Relman DA. Incomplete recovery and individualized responses of the human distal gut microbiota to repeated antibiotic perturbation. Proc Natl Acad Sci U S A 2011;108 Suppl 1:45544561.

21. Correale J, Farez M. Association between parasite infection and immune responses in multiple sclerosis. Ann Neurol 2007;61:97108.

22. Büning J, Homann N, von Smolinski D, et al. Helminths as governors of inflammatory bowel disease. Gut 2008;57:1182-1183.

23. Elliott DE, Summers RW, Weinstock JV. Helminths as governors of immune-mediated inflammation. Int J Parasitol 2007;37:457-464.

24. Weinstock JV, Summers RW, Elliott DE. Role of helminths in regulating mucosal inflammation. Springer Semin Immunopathol 2005;27:249-271.
25. Jostins L, Ripke S, Weersma RK, et al. Host-microbe interactions have shaped the genetic architecture of inflammatory bowel disease. Nature 2012;491:119-124.

26. Liu JZ, van Sommeren S, Huang $\mathrm{H}$, et al. Association analyses identify 38 susceptibility loci for inflammatory bowel disease and highlight shared genetic risk across populations. Nat Genet 2015;47:979-986

27. D'Haens GR, Geboes K, Peeters M, Baert F, Penninckx F, Rutgeerts P. Early lesions of recurrent Crohn's disease caused by infusion of intestinal contents in excluded ileum. Gastroenterology 1998;114:262-267.

28. Macpherson A, Khoo UY, Forgacs I, Philpott-Howard J, Bjarnason I. Mucosal antibodies in inflammatory bowel disease are directed against intestinal bacteria. Gut 1996;38:365-375.

29. Sokol H, Seksik P. The intestinal microbiota in inflammatory bowel diseases: time to connect with the host. Curr Opin Gastroenterol 2010;26:327-331

30. Sokol H, Pigneur B, Watterlot L, et al. Faecalibacterium prausnitzii is an anti-inflammatory commensal bacterium identified by gut microbiota analysis of Crohn disease patients. Proc Natl Acad Sci U S A 2008;105:16731-16736.

31. Varela E, Manichanh C, Gallart M, et al. Colonisation by Faecalibacterium prausnitzii and maintenance of clinical remission in patients with ulcerative colitis. Aliment Pharmacol Ther 2013;38:151-161.

32. Roediger WE, Moore J, Babidge W. Colonic sulfide in pathogenesis and treatment of ulcerative colitis. Dig Dis Sci 1997;42:1571-1579.

33. Gevers D, Kugathasan S, Denson LA, et al. The treatment-naive microbiome in new-onset Crohn's disease. Cell Host Microbe 2014;15:382-392.

34. Morgan XC, Tickle TL, Sokol H, et al. Dysfunction of the intestinal microbiome in inflammatory bowel disease and treatment. Genome Biol 2012;13:R79.

35. Morgan XC, Kabakchiev B, Waldron L, et al. Associations between host gene expression, the mucosal microbiome, and clinical outcome in the pelvic pouch of patients with inflammatory bowel disease. Genome Biol 2015;16:67.

36. Atarashi K, Tanoue T, Oshima K, et al. Treg induction by a rationally selected mixture of Clostridia strains from the human microbiota. Nature 2013;500:232-236.

37. Bennet JD, Brinkman M. Treatment of ulcerative colitis by implantation of normal colonic flora. Lancet 1989;1:164.

38. Colman RJ, Rubin DT. Fecal microbiota transplantation as therapy for inflammatory bowel disease: a systematic review and metaanalysis. J Crohns Colitis 2014;8:1569-1581.

39. Moayyedi P, Surette MG, Kim PT, et al. Fecal microbiota transplantation induces remission in patients with active ulcerative colitis in a randomized controlled trial. Gastroenterology 2015; 149:102-109.e6.

40. Rossen NG, Fuentes S, van der Spek MJ, et al. Findings from a randomized controlled trial of fecal transplantation for patients with ulcerative colitis. Gastroenterology 2015;149:110-118.e4. 